\title{
Interference coordination in full-duplex HetNet with large-scale antenna arrays*
}

\author{
Zhao-yang ZHANG \\ (College of Information Science and Electronic Engineering, Zhejiang University, Hangzhou 310027, China) \\ E-mail: ning_ming@zju.edu.cn; levy_lv@zju.edu.cn
}

Received Jan. 15, 2017; Revision accepted Apr. 6, 2017; Crosschecked June 5, 2017

\begin{abstract}
Massive multiple-input multiple-output (MIMO), small cell, and full-duplex are promising techniques for future $5 \mathrm{G}$ communication systems, where interference has become the most challenging issue to be addressed. In this paper, we provide an interference coordination framework for a two-tier heterogeneous network (HetNet) that consists of a massive-MIMO enabled macro-cell base station (MBS) and a number of full-duplex small-cell base stations (SBSs). To suppress the interferences and maximize the throughput, the full-duplex mode of each SBS at the wireless backhaul link (i.e., in-band or out-of-band), which has a different impact on the interference pattern, should be carefully selected. To address this problem, we propose two centralized algorithms, a genetic algorithm (GEA) and a greedy algorithm (GRA). To sufficiently reduce the computational overhead of the MBS, a distributed graph coloring algorithm (DGCA) based on price is further proposed. Numerical results demonstrate that the proposed algorithms significantly improve the system throughput.
\end{abstract}

Key words: Massive MIMO; Full-duplex; Small cell; Wireless backhaul; Distributed algorithm http://dx.doi.org/10.1631/FITEE.1700047

CLC number: TN929.5

\section{Introduction}

The 5th-generation (5G) communication system has drawn more and more research interest recently. Compared with the 4th-generation (4G) communication systems, i.e., the 3rd Generation Partnership Project (3GPP) Long Term Evolution Advanced (LTE-A) standard (3GPP, 2012a), the most challenging problem in $5 \mathrm{G}$ is to achieve substantially higher throughput. As such, a small-cell heterogeneous network (HetNet), where small cells provide local capacity enhancements (e.g., hotspots in urban areas) and macro cells support highly mobile terminals, has been proposed as a promising technique for $5 \mathrm{G}$ due to its high spatial reuse gains (Hoydis

$\ddagger$ Corresponding author

* Project supported by the National High-Tech R\&D Program (863) of China (No. 2014AA01A702) and the National Natural Science Foundation of China (No. 61371094)

(1) ORCID: Zhao-yang ZHANG, http://orcid.org/0000-0003-23466228

(C)Zhejiang University and Springer-Verlag Berlin Heidelberg 2017 et al., 2011; Boccardi et al., 2014). However, the inherent co-tier and cross-tier interferences in HetNets result in serious degradation to the overall throughput performance. Thus, suppressing these interferences in small-cell HetNets is an emerging issue to be addressed.

To address this issue, two main approaches have been proposed in previous papers. On the one hand, massive multiple-input multiple-output (MIMO) techniques were proposed to reduce the interference. As demonstrated in Marzetta (2010), Rusek et al. (2013), and Larsson et al. (2014), massive MIMO scales up the conventional MIMO by orders of magnitude and exploits the additional spatial degrees of freedom (DoFs), thus providing aggressive spatial multiplexing capabilities and significant array gains. On these bases, Hoydis et al. (2013) proposed a network framework where macro-cell base stations (MBSs) with large-scale antenna arrays provide services for highly mobile user equipment (UE) 
and dense small cells support UE with low mobility. Simulation results showed that the small-cell HetNet with massive MIMO could achieve very high throughput. A similar framework was invoked in Hosseini et al. (2013), in which a covariance-based precoding scheme was employed for interference cancellation at the price of sacrificing excess antennas.

On the other hand, full-duplex could be used for interference coordination. By transmitting and receiving signals on the same frequency at the same time (Choi et al., 2010; Sabharwal et al., 2014; Liu et al., 2015; Thilina et al., 2015; Bharadia and Katti, 2016), full-duplex techniques could help small cells efficiently reuse the radio access network (RAN) spectrum (Goyal et al., 2013). In Goyal et al. (2014), an uplink/downlink user scheduling scheme that maximizes the overall utility of small-cell users was developed to investigate the feasibility conditions of full-duplex operations. In Li et al. (2015), three strategies of small-cell in-band wireless backhaul with massive MIMO were provided and analyzed, and simulation results demonstrated that applying full-duplex techniques at small cells could provide larger gain. Tabassum et al. (2016) considered the backhaul links in both in- and out-of-band fullduplex modes, and derived the optimal mode selection proportion of small-cell base stations (SBSs) for special cases to maximize the outage capacity.

Although the feasibility of combining massive MIMO, small cell, and full-duplex has been studied in the above works, comprehensive analyses of how to effectively suppress the co-tier interference and cross-tier interference in such scenarios are still absent, and we attempt to fill that gap in this study. In this paper, the downlink of a two-tier HetNet with a massive MIMO-enabled MBS and a number of full-duplex SBSs working in in-band or out-ofband wireless backhaul, respectively, is considered. With full consideration of the different impacts of the full-duplex modes, the co-tier and cross-tier interferences are thoroughly analyzed. To maximize the overall system throughput, we propose centralized algorithms and a distributed algorithm to decide the full-duplex mode selection strategies of SBSs.

\section{System model}

In this study, we consider a downlink two-tier HetNet as illustrated in Fig. 1. An existing MBS

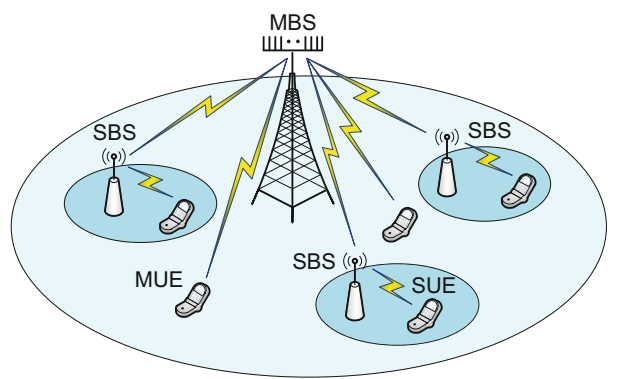

Fig. 1 A two-tier HetNet system with one massive MIMO macro-cell base station and $S$ full-duplex small-cell base stations

comprising $N$ transmit antennas provides wireless services for $K$ single-antenna macro-cell UE (MUE). Additionally, there exist $S$ single-antenna SBSs in the cell, the MBS provides wireless backhaul connections to these SBSs, and each SBS could provide service for a single-antenna small-cell UE (SUE) simultaneously using full-duplex techniques.

Massive MIMO is invoked by the MBS, which refers to the case where $N \gg K$ and $N \gg S$. Linear zero-forcing beamforming (LZFBF) with equal power per stream is employed in the MBS access links to MUE and wireless backhauls to SBSs. Moreover, we assume the wireless channels are subject to pathloss, shadowing, and Rayleigh flat fading throughout the paper. For analytical convenience, we further assume that the transmissions across the tiers are synchronized perfectly, and the perfect channel state information (CSI) can be obtained by the MBS from uplink training. The MBS can then use it to design the downlink precoder, which is facilitated by considering time division duplexing (TDD) at the MBS such that the channel reciprocity is guaranteed. Note that although we restrict our attention to the downlink case, the proposed algorithms are applicable to the uplink scenarios with some minor modifications.

\subsection{Full-duplex modes of SBSs}

Before analyzing the macro- and small-cell transmissions, let us look into the full-duplex modes of each SBS. As stated in Tabassum et al. (2016), each SBS can operate in two modes for wireless backhaul transmission: (1) out-of-band full-duplex mode (OBFD), in which the access link and backhaul link are conducted in orthogonal channels; (2) in-band full-duplex mode (IBFD), in which the access link and backhaul link are conducted at the same frequency band simultaneously. 
Because the MBS provides services for MUE and SBSs at the same time, assigning these two links with identical frequency band results in a complicated transmission coordination problem. As such, we divide the total available bandwidth into two equal parts, denoted as $F_{1}$ and $F_{2}$, and assume that the macro-cell access link exploits the $F_{1}$ band while the backhaul link occupies $F_{2}$. In this setting, the choice between $F_{1}$ and $F_{2}$ for the small-cell access link directly determines the full-duplex mode of the corresponding SBS. If small-cell access link employs $F_{2}$, then this SBS is in IBFD mode, and vice versa. For notational convenience, we refer to the set of all IBFD SBSs as $\mathcal{S}_{\text {I }}$ and that of all OBFD SBSs as $\mathcal{S}_{\mathrm{O}}$. The set of all SBSs is denoted as $\mathcal{S}$. From Fig. 2, we see that SBSs in different sets experience distinct interference patterns. Keeping these two interference patterns in mind, we are able to model the considered HetNet in detail.

\subsection{Macro-cell transmission model}

First, we investigate the macro-cell transmissions, i.e., the access links from the MBS to the MUE. According to Fig. 2a, the $k$ th MUE experiences interference from SBSs in $\mathcal{S}_{\mathrm{O}}$, so the received signal at the $k$ th MUE can by modeled by

$$
\begin{aligned}
y_{\mathrm{MUE}, k}(t)= & \boldsymbol{h}_{\mathrm{b} 2 \mathrm{mu}, k} \boldsymbol{W} \boldsymbol{x}_{\mathrm{b} 2 \mathrm{mu}}(t) \\
& +\sum_{i \in \mathcal{S}_{\mathrm{O}}} h_{\mathrm{s} 2 \mathrm{mu}, i k} x_{\mathrm{s} 2 \mathrm{su}, i}(t)+n(t),
\end{aligned}
$$

where $\boldsymbol{h}_{\mathrm{b} 2 \mathrm{mu}, k} \in \mathbb{C}^{1 \times N}$ denotes the fading channel from the MBS to the $k$ th MUE, $\boldsymbol{W} \in \mathbb{C}^{N \times K}$ is the downlink precoder, $\boldsymbol{x}_{\mathrm{b} 2 \mathrm{mu}}(t) \sim \mathcal{C N}\left(\mathbf{0},\left(P_{\mathrm{M}} / K\right) \boldsymbol{I}_{K}\right)$

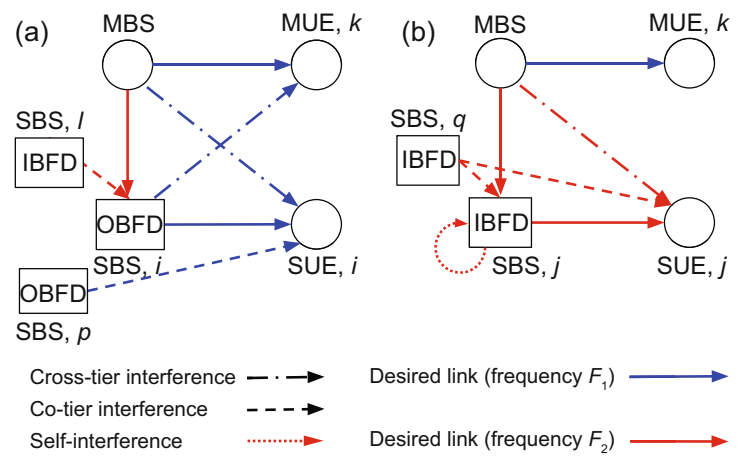

Fig. 2 Graphical illustration of the different interference in OBFD (a) and IBFD (b) modes. Downlink cross- and co-tier interferences experienced at MUE, SBS, and SUE are illustrated (References to color refer to the online version of this figure) represents the $K \times 1$ symbol vector, and $P_{\mathrm{M}}$ is the transmit power of the MUE assigned to each frequency band. In additioin, $h_{\mathrm{s} 2 \mathrm{mu}, i k}$ indicates the channel from the $i$ th SBS to the $k$ th MUE, $x_{\mathrm{s} 2 \mathrm{su}, i}(t) \sim \mathcal{C} \mathcal{N}\left(0, P_{\mathrm{S}}\right)$ is the symbol transmitted by the $i$ th SBS, and $P_{\mathrm{S}}$ denotes the transmit power of each SBS. Finally, $n(t)$ stands for the complex Gaussian noise with mean zero and variance $\sigma^{2}$. Based on this model, the SINR at the $k$ th MUE can be simply obtained by

$$
\gamma_{\mathrm{MUE}, k}=\frac{P_{\mathrm{M}}\left\|\boldsymbol{h}_{\mathrm{b} 2 \mathrm{mu}, k} \boldsymbol{W}\right\|^{2} / K}{P_{\mathrm{S}} \sum_{i \in \mathcal{S}_{\mathrm{O}}} h_{\mathrm{s} 2 \mathrm{mu}, i k}^{2}+\sigma^{2}} .
$$

\subsection{Small-cell transmission model}

Recalling that different full-duplex modes induce distinct interference patterns, we need to model them separately.

\subsubsection{SBS in OBFD mode}

1. Backhaul link: As shown in Fig. 2a, the $i$ th SBS operates in OBFD mode and suffers from co-tier interference from SBSs in $\mathcal{S}_{\text {I }}$. As such, the received signal at the $i$ th SBS can be expressed as

$$
\begin{aligned}
y_{\mathrm{SBS}, i}^{\mathrm{O}}(t)= & \boldsymbol{h}_{\mathrm{b} 2 \mathrm{~s}, i} \boldsymbol{G} \boldsymbol{x}_{\mathrm{b} 2 \mathrm{~s}}(t) \\
& +\sum_{l \in \mathcal{S}_{\mathrm{I}}} h_{\mathrm{s} 2 \mathrm{~s}, i l} x_{\mathrm{s} 2 \mathrm{su}, l}(t)+n(t),
\end{aligned}
$$

where $\boldsymbol{h}_{\mathrm{b} 2 \mathrm{~s}, i} \in \mathbb{C}^{1 \times N}$ denotes the channel matrix from the MBS to the $i$ th SBS, $G$ is the precoding matrix, $\boldsymbol{x}_{\mathrm{b} 2 \mathrm{~s}}(t) \in \mathcal{C} \mathcal{N}\left(\mathbf{0},\left(P_{\mathrm{M}} / S\right) \boldsymbol{I}_{S}\right)$ is the $S \times 1$ symbol vector from the MBS to SBSs. $h_{\mathrm{s} 2 \mathrm{~s}, i l}$ represents the channel between the $i$ th SBS and the lth SBS, which incurs the co-tier interference. Thereby, the $i$ th SBS in OBFD mode has the SINR

$$
\gamma_{\mathrm{SBS}, i}^{\mathrm{O}}=\frac{\frac{P_{\mathrm{M}}}{S}\left\|\boldsymbol{h}_{\mathrm{b} 2 \mathrm{~s}, i} \boldsymbol{G}\right\|^{2}}{P_{\mathrm{S}} \sum_{l \in \mathcal{S}_{\mathrm{I}}} h_{\mathrm{s} 2 \mathrm{~s}, i l}^{2}+\sigma^{2}} .
$$

2. Access link: As illustrated in Fig. 2a, the $i$ th SUE suffers from interference from the MBS and the other SBSs in OBFD mode, and thus it suffers from both co-tier interference and cross-tier interference. The received signal can be expressed as

$$
\begin{aligned}
y_{\mathrm{SUE}, i}^{\mathrm{O}}(t)= & h_{\mathrm{s} 2 \mathrm{su}, i i} x_{\mathrm{s} 2 \mathrm{su}, i}(t)+\sum_{p \in \mathcal{S}_{\mathrm{O}} \backslash i} h_{\mathrm{s} 2 \mathrm{su}, i p} x_{\mathrm{s} 2 \mathrm{su}, p}(t) \\
& +\boldsymbol{h}_{\mathrm{b} 2 \mathrm{su}, i} \boldsymbol{W} \boldsymbol{x}_{\mathrm{b} 2 \mathrm{mu}}(t)+n(t),
\end{aligned}
$$


where $h_{\mathrm{s} 2 \mathrm{su}, i i}$ denotes the channel from the $i$ th OBFD SBS to its associated SUE, and $h_{\mathrm{s} 2 \mathrm{su}, i p}$ denotes the channel between the $p$ th SBS and the $i$ th SUE, which incurs the co-tier interference. $\boldsymbol{h}_{\mathrm{b} 2 \mathrm{su}, i} \in$ $\mathbb{C}^{1 \times N}$ is the channel matrix from the MBS to the $i$ th SUE, which incurs the cross-tier interference.

To mitigate the cross-tier interference from the MBS, we exploit the modified zero-forcing precoder proposed in Li et al. (2015), which is

$$
\boldsymbol{W}=\boldsymbol{R} \cdot\left(\boldsymbol{h}_{\mathrm{b} 2 \mathrm{mu}} \boldsymbol{R}\right)^{+} \cdot \boldsymbol{\Gamma}_{\boldsymbol{W}}^{0.5},
$$

where $\boldsymbol{h}_{\mathrm{b} 2 \mathrm{mu}} \triangleq\left[\boldsymbol{h}_{\mathrm{b} 2 \mathrm{mu}, 1}^{\mathrm{H}}, \boldsymbol{h}_{\mathrm{b} 2 \mathrm{mu}, 2}^{\mathrm{H}}, \cdots, \boldsymbol{h}_{\mathrm{b} 2 \mathrm{mu}, K}^{\mathrm{H}}\right]^{\mathrm{H}}$ is the $K \times N$ channel matrix from the MBS to MUE, $\boldsymbol{R} \in \mathbb{C}^{N \times(N-S)}$ is a matrix that satisfies $\boldsymbol{h}_{\mathrm{b} 2 \mathrm{su}} \boldsymbol{R}=\mathbf{0}_{S \times(N-S)}$, and $\boldsymbol{h}_{\mathrm{b} 2 \mathrm{su}} \triangleq$ $\left[\boldsymbol{h}_{\mathrm{b} 2 \mathrm{su}, 1}^{\mathrm{H}}, \boldsymbol{h}_{\mathrm{b} 2 \mathrm{su}, 2}^{\mathrm{H}}, \cdots, \boldsymbol{h}_{\mathrm{b} 2 \mathrm{su}, S}^{\mathrm{H}}\right]^{\mathrm{H}}$ represents the crosstier interference channel matrix. $\boldsymbol{h}^{+}=\boldsymbol{h}^{\mathrm{H}}\left(\boldsymbol{h} \boldsymbol{h}^{\mathrm{H}}\right)$ calculates the Moore-Penrose pseudo-inverse of $\boldsymbol{h} . \boldsymbol{\Gamma}_{\boldsymbol{W}}^{0.5}$ is a diagonal matrix that normalizes $\boldsymbol{R}\left(\boldsymbol{h}_{\mathrm{b} 2 \mathrm{mu}} \boldsymbol{R}\right)^{+}$.

Thus, the SINR of the $i$ th SUE can be obtained by

$$
\gamma_{\mathrm{SUE}, i}^{\mathrm{O}}=\frac{P_{\mathrm{S}} h_{\mathrm{s} 2 \mathrm{su}, i i}^{2}}{P_{\mathrm{S}} \sum_{p \in \mathcal{S}_{\mathrm{O}} \backslash i} h_{\mathrm{s} 2 \mathrm{su}, i p}^{2}+\sigma^{2}} .
$$

\subsubsection{SBS in IBFD mode}

1. Backhaul link: For IBFD SBS $j$, the co-tier interference and self-interference should be considered (Fig. 2b). Specifically, the received signal at the $j$ th SBS is given by

$$
\begin{aligned}
y_{\mathrm{SBS}, j}^{\mathrm{I}}(t)= & \boldsymbol{h}_{\mathrm{b} 2 \mathrm{~s}, j} \boldsymbol{G} \boldsymbol{x}_{\mathrm{b} 2 \mathrm{~s}}(t)+\sum_{q \in \mathcal{S}_{\mathrm{I}} \backslash j} h_{\mathrm{s} 2 \mathrm{~s}, j q} x_{\mathrm{s} 2 \mathrm{su}, q}(t) \\
& +\sqrt{I_{\mathrm{SI}}} w(t)+n(t),
\end{aligned}
$$

where $\sqrt{I_{\mathrm{SI}}} w(t)$ denotes the residual self-interference and $E\left[\|w(t)\|^{2}\right]=1$. Based on Tabassum et al. (2016), $I_{\mathrm{SI}}$ can be modeled as $I_{\mathrm{SI}}=P_{\mathrm{S}} / \rho$, where $\rho$ represents the self-interference cancellation capability. Therefore, the SINR at the $j$ th SBS in IBFD mode is obtained by

$$
\gamma_{\mathrm{SBS}, j}^{\mathrm{I}}=\frac{P_{\mathrm{M}}\left\|\boldsymbol{h}_{\mathrm{b} 2 \mathrm{~s}, j} \boldsymbol{G}\right\|^{2} / S}{P_{\mathrm{S}} \sum_{q \in \mathcal{S}_{\mathrm{I}} \backslash j} h_{\mathrm{s} 2 \mathrm{~s}, j q}^{2}+I_{\mathrm{SI}}+\sigma^{2}} .
$$

2. Access link: As illustrated in Fig. 2b, the $j$ th SUE suffers from cross-tier interference from the MBS and co-tier interference from other SBSs in IBFD mode, thus inducing the received signal:

$$
\begin{aligned}
y_{\mathrm{SUE}, j}^{\mathrm{I}}(t)= & h_{\mathrm{s} 2 \mathrm{su}, j j} x_{\mathrm{s} 2 \mathrm{su}, j}(t)+\sum_{q \in \mathcal{S}_{\mathrm{I}} \backslash j} h_{\mathrm{s} 2 \mathrm{su}, j q} x_{\mathrm{s} 2 \mathrm{su}, q}(t) \\
& +\boldsymbol{h}_{\mathrm{b} 2 \mathrm{su}, j} \boldsymbol{G} \boldsymbol{x}_{\mathrm{b} 2 \mathrm{~s}}(t)+n(t) .
\end{aligned}
$$

Again we invoke the results in Li et al. (2015) to remove the cross-tier interference. In this sense, $G$ is given by

$$
\boldsymbol{G}=\boldsymbol{R} \cdot\left(\boldsymbol{h}_{\mathrm{b} 2 \mathrm{~s}} \boldsymbol{R}\right)^{+} \cdot \boldsymbol{\Gamma}_{\boldsymbol{G}}^{0.5}
$$

where $\boldsymbol{h}_{\mathrm{b} 2 \mathrm{~s}}=\left[\boldsymbol{h}_{\mathrm{b} 2 \mathrm{~s}, 1}^{\mathrm{H}}, \boldsymbol{h}_{\mathrm{b} 2 \mathrm{~s}, 2}^{\mathrm{H}}, \cdots, \boldsymbol{h}_{\mathrm{b} 2 \mathrm{~s}, S}^{\mathrm{H}}\right]^{\mathrm{H}}$ represents the channel matrix from MBS to SBSs, and $\Gamma_{G}^{0.5}$ is a diagonal matrix that normalizes $\boldsymbol{R}\left(\boldsymbol{h}_{\mathrm{b} 2 \mathrm{~s}} \boldsymbol{R}\right)^{+}$. Then, the SINR of the $j$ th SUE can be derived as

$$
\gamma_{\mathrm{SUE}, j}^{\mathrm{I}}=\frac{P_{\mathrm{S}} h_{\mathrm{s} 2 \mathrm{su}, j j}^{2}}{P_{\mathrm{S}} \sum_{q \in \mathcal{S}_{\mathrm{I}} \backslash j} h_{\mathrm{s} 2 \mathrm{su}, j q}^{2}+\sigma^{2}} .
$$

\section{Problem formulation}

Due to the different interference patterns of these two full-duplex modes, we should reasonably assign the full-duplex modes to each SBS to maximize the overall throughput of this two-tier HetNet. In this section, we formulate the full-duplex mode selection problem as an optimization problem.

Based on Shannon theory and the SINR results derived in Section 2, we can obtain the overall throughput as follows:

$$
\begin{aligned}
C= & C_{\mathrm{M}}+C_{\mathrm{S}}^{\mathrm{O}}+C_{\mathrm{S}}^{\mathrm{I}} \\
= & \sum_{k \in \mathcal{K}} \frac{1}{2} \log _{2}\left(1+\gamma_{\mathrm{MUE}, k}\right) \\
& +\sum_{i \in \mathcal{S}_{\mathrm{O}}} \frac{1}{2} \log _{2}\left(1+\min \left(\gamma_{\mathrm{SBS}, i}^{\mathrm{O}}, \gamma_{\mathrm{SUE}, i}^{\mathrm{O}}\right)\right) \\
& +\sum_{j \in \mathcal{S}_{\mathrm{I}}} \frac{1}{2} \log _{2}\left(1+\min \left(\gamma_{\mathrm{SBS}, j}^{\mathrm{I}}, \gamma_{\mathrm{SUE}, j}^{\mathrm{I}}\right)\right),
\end{aligned}
$$

where $C_{\mathrm{M}}, C_{\mathrm{S}}^{\mathrm{O}}$, and $C_{\mathrm{S}}^{\mathrm{I}}$ denote the throughput of the macro-cell tier, small-cell tier in the OBFD mode, and small-cell tier in the IBFD mode, respectively, and $\mathcal{K}$ is the set of MUEs.

For ease of exposition, an indicator vector $\boldsymbol{x} \triangleq$ $\left[x_{1}, x_{2}, \cdots, x_{S}\right]$ is used to determine the full-duplex mode of each SBS, i.e.,

$$
x_{i}= \begin{cases}1, & \text { the } i \text { th SBS is in IBFD mode } \\ 0, & \text { the } i \text { th SBS is in OBFD mode }\end{cases}
$$

Based on this definition, the throughput maximization problem can be derived as problem (15), by substituting Eqs. (2), (4), (7), (9), and (12) into 


$$
\begin{aligned}
\max _{\boldsymbol{x}} C= & \sum_{k \in \mathcal{K}} \log _{2}\left(1+\frac{1}{\sum_{i \in \mathcal{S}}\left(1-x_{i}\right) \alpha_{k, i}+\beta_{k}}\right) \\
& +\sum_{i \in \mathcal{S}}\left(1-x_{i}\right) \log _{2}\left(1+\min \left(\frac{1}{\sum_{j \in \mathcal{S}} x_{j} a_{i, j}+b_{i}}, \frac{1}{\sum_{j \in \mathcal{S}}\left(1-x_{j}\right) c_{i, j}+d_{i}}\right)\right) \\
& +\sum_{i \in \mathcal{S}} x_{i} \log _{2}\left(1+\min \left(\frac{1}{\sum_{j \in \mathcal{S}} x_{j} e_{i, j}+f_{i}}, \frac{1}{\sum_{j \in \mathcal{S}} x_{j} g_{i, j}+h_{i}}\right)\right)
\end{aligned}
$$

s.t. $x_{i} \in\{0,1\}, \forall i \in \mathcal{S}$.

Eq. (13), where

$$
\begin{aligned}
& \alpha_{k, i}=\frac{K P_{\mathrm{S}} h_{\mathrm{s} 2 \mathrm{mu}, i k}^{2}}{P_{\mathrm{M}}\left\|\boldsymbol{h}_{\mathrm{b} 2 \mathrm{mu}, k} \boldsymbol{W}\right\|^{2}}, \beta_{k}=\frac{K \sigma^{2}}{P_{\mathrm{M}}\left\|\boldsymbol{h}_{\mathrm{b} 2 \mathrm{mu}, k} \boldsymbol{W}\right\|^{2}}, \\
& a_{i, j}=\frac{S P_{\mathrm{S}} h_{\mathrm{s} 2 \mathrm{~s}, i j}^{2}}{P_{\mathrm{M}}\left\|\boldsymbol{h}_{\mathrm{b} 2 \mathrm{~s}, i} \boldsymbol{G}\right\|^{2}}, \quad b_{i}=\frac{S \sigma^{2}}{P_{\mathrm{M}}\left\|\boldsymbol{h}_{\mathrm{b} 2 \mathrm{~s}, i} \boldsymbol{G}\right\|^{2}}, \\
& c_{i, j}=\left\{\begin{array}{ll}
h_{\mathrm{s} 2 \mathrm{su}, i j}^{2} / h_{\mathrm{s} 2 \mathrm{su}, i i}^{2}, & j \neq i, \\
0, & j=i,
\end{array} \quad d_{i}=\frac{\sigma^{2}}{P_{\mathrm{S}} h_{\mathrm{s} 2 \mathrm{su}, i i}^{2}},\right. \\
& e_{i, j}=\left\{\begin{array}{ll}
\frac{S P_{\mathrm{S}} h_{\mathrm{s} 2 \mathrm{~s}, i j}^{2}}{P_{\mathrm{M}}\left\|\boldsymbol{h}_{\mathrm{b} 2 \mathrm{~s}, i} \boldsymbol{G}\right\|^{2}}, & j \neq i, \\
0, & j=i,
\end{array} f_{i}=\frac{S\left(I_{\mathrm{SI}}+\sigma^{2}\right)}{P_{\mathrm{M}}\left\|\boldsymbol{h}_{\mathrm{b} 2 \mathrm{~s}, i} \boldsymbol{G}\right\|^{2}},\right. \\
& g_{i, j}=\left\{\begin{array}{ll}
h_{\mathrm{s} 2 \mathrm{su}, i j}^{2} / h_{\mathrm{s} 2 \mathrm{su}, i i}^{2}, & j \neq i, \\
0, & j=i,
\end{array} \quad h_{i}=\frac{\sigma^{2}}{P_{\mathrm{S}} h_{\mathrm{s} 2 \mathrm{su}, i i}^{2}} .\right.
\end{aligned}
$$

The problem we formulate is a combinatorial optimization problem. The conventional brute-force algorithm has the complexity $\mathcal{O}\left(2^{S}\right)$, incurring unacceptable computational overhead for large $S$, i.e., highly dense small-cell networks. To solve problem (15) efficiently, we further assume as in Tabassum et al. (2016) that the throughput of the backhaul link is always larger than that of the small-cell access link. This assumption is practical due to the performance gain provided by massive MIMO and the advanced self-interference cancellation techniques in full-duplex systems (Jain et al., 2011). Under this condition, we recast problem (15) as

$$
\begin{aligned}
\max _{\boldsymbol{x}} C & =\sum_{k \in \mathcal{K}} \log _{2}\left(1+\frac{1}{\sum_{i \in \mathcal{S}}\left(1-x_{i}\right) \alpha_{k, i}+\beta_{k}}\right) \\
& +\sum_{i \in \mathcal{S}}\left(1-x_{i}\right) \log _{2}\left(1+\frac{1}{\sum_{j \in \mathcal{S}}\left(1-x_{j}\right) c_{i, j}+d_{i}}\right) \\
& +\sum_{i \in \mathcal{S}} x_{i} \log _{2}\left(1+\frac{1}{\sum_{j \in \mathcal{S}} x_{j} c_{i, j}+d_{i}}\right) \\
\text { s.t. } & x_{i} \in\{0,1\}, \forall i \in \mathcal{S} .
\end{aligned}
$$

For notational convenience, we have replaced $g_{i, j}$ and $h_{i}$ in the third term with $c_{i, j}$ and $d_{i}$, respectively. This is practical because $c_{i, j}=g_{i, j}$ and $d_{i}=h_{i}$.

\section{Interference coordination with cen- tralized algorithms}

Obviously, problem (16) is still a combinatorial optimization problem. Also, since the objective function is nonconvex, the optimal vector $\boldsymbol{x}$ can hardly be obtained even if we relax the constraint $x_{i} \in\{0,1\}$. As such, the proper near-optimal solutions are desired. In this section, we propose two centralized algorithms, i.e., a genetic algorithm and a greedy algorithm, so that the MBS collects the global CSI and acts as a central scheduler.

\subsection{Genetic algorithm}

The genetic algorithm (GEA) is an adaptive heuristic search algorithm based on the evolutionary ideas of natural selection and genetics; it represents an intelligent exploitation of a random search to solve optimization problems. Additionally, in searching a large state-space, GEA may offer significant benefits over other typical optimization techniques. As such, in this subsection, we apply GEA to our optimization problem.

Because GEA is a mature algorithm, the theory will not be elaborated specifically in this paper; instead, we directly list the procedure in Algorithm 1. In the settings of key parameters (e.g., the maximum number of iterations, the probability of crossover and mutation), we adopt some general values based on experience. Notably, it is necessary to give serious consideration to the setting of population size, because if we set the value as a constant, the performance of GEA will significantly degrade when the 
value of $S$ is much larger than the population size; thus, in this study, we set it as $S+10$, a variable depending on $S$.

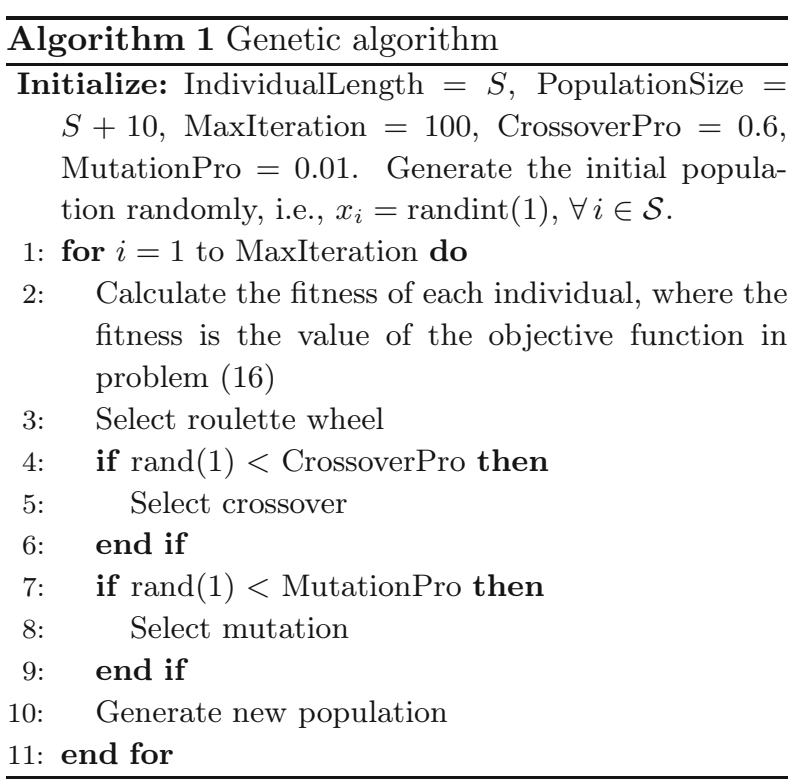

As shown in Algorithm 1, the complexity of GEA depends mainly on step 5. We can easily verify that the complexity of calculating the objective function in problem (16) for one individual is $\mathcal{O}\left(S^{2}\right)$, and thus step 4 needs complexity of $\mathcal{O}\left(S^{3}\right)$. Moreover, because the maximum number of iterations we set is a constant, the overall complexity of GEA is still $\mathcal{O}\left(S^{3}\right)$.

\subsection{Greedy algorithm}

The greedy algorithm (GRA) is an algorithmic paradigm that follows the problem-solving heuristic of making the locally optimal choice at each stage. In many combinatorial optimization problems, e.g., traveling salesman problem (TSP), a greedy strategy is often used as an effective method to find an available solution. In this subsection, a greedy algorithm is proposed to solve our problem.

As shown in Algorithm 2, the basic idea of GRA is that at each iteration we switch the mode of an SBS when this switch can bring the largest throughput gain; the iteration will terminate if the largest throughput gain becomes negative. Obviously, GRA can make the best choice at each iteration; however, it may fail to produce the optimal solution because it is short-sighted. We also note that the solutions obtained by GRA will be influenced by the random initial solutions; thus, to obtain the optimal solution as often as possible, we should generate a lot of initial solutions and choose the one that has the best performance. As for the complexity of GRA, we can see that in Algorithm 2, the complexity of step 4 is $\mathcal{O}(S)$ and the complexity of step 7 is $\mathcal{O}\left(S^{2}\right)$, and thus the complexity of one iteration is $\mathcal{O}\left(S^{3}\right)$, but its rate of convergence cannot be promised because it depends on the quality of the initial random solutions. We can determine only that the overall complexity of GRA will be no less than $\mathcal{O}\left(S^{3}\right)$. However, we can evaluate the convergence rate of GRA by comparing its computation time with that of GEA, because they share the same iteration complexity, i.e., $\mathcal{O}\left(S^{3}\right)$. The comparison is shown in Section 6.

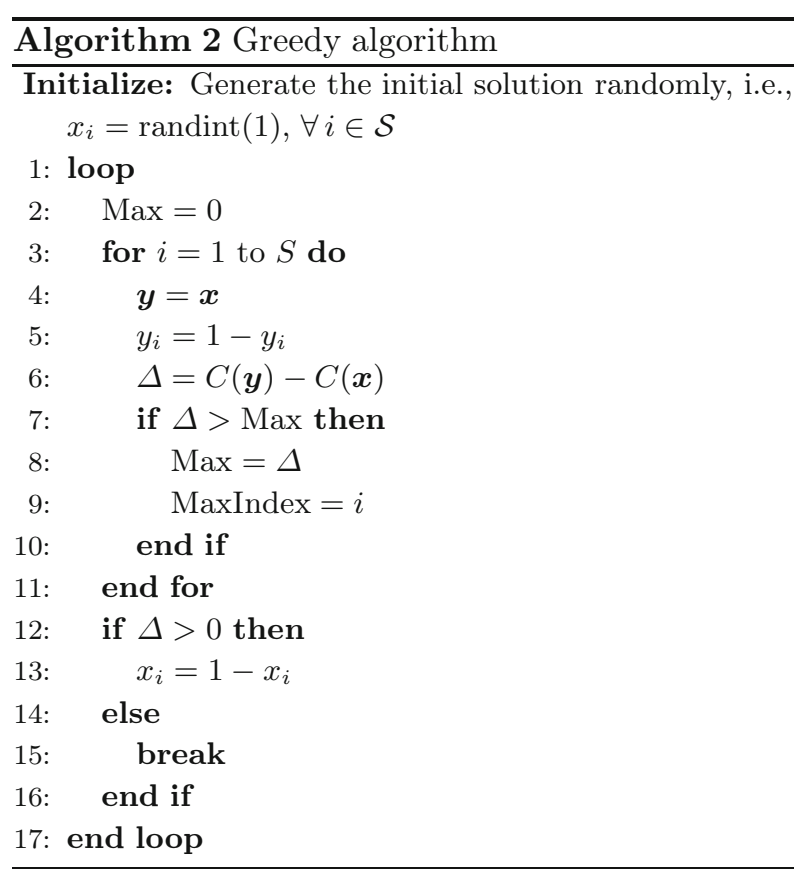

For the iterative process in the proposed GRA, we prove its convergence in the following proposition:

Proposition 1 For any initial solution, GRA can converge.

Proof We denote the number of iterations as $t=1,2, \cdots$, and the iterative procedure of GRA is in fact a sequence of $C_{t}$. As described in Algorithm 2, we always promise that $\Delta>0$, i.e., $C_{t+1}>C_{t}$; thus, $C_{t}$ is monotonously increasing with $t$. Also, we can 
prove that $C_{t}$ has an upper bound as follows:

$$
\begin{aligned}
C & =\sum_{k \in \mathcal{K}} \log _{2}\left(1+\frac{1}{\sum_{i \in \mathcal{S}}\left(1-x_{i}\right) \alpha_{k, i}+\beta_{k}}\right) \\
& +\sum_{i \in \mathcal{S}}\left(1-x_{i}\right) \log _{2}\left(1+\frac{1}{\sum_{j \in \mathcal{S}}\left(1-x_{j}\right) c_{i, j}+d_{i}}\right) \\
& +\sum_{i \in \mathcal{S}} x_{i} \log _{2}\left(1+\frac{1}{\sum_{j \in \mathcal{S}} x_{j} c_{i, j}+d_{i}}\right) \\
& <\sum_{k \in \mathcal{K}} \log _{2}\left(1+\frac{1}{\beta_{k}}\right)+\sum_{i \in \mathcal{S}} \log _{2}\left(1+\frac{1}{d_{i}}\right)=U,
\end{aligned}
$$

where $U$ is a constant that depends on $\beta_{k}$ and $d_{i}$, which are limited by CSI, noise variance, and transmit power.

Because $C_{t}$ is monotonously increasing with $t$ and has an upper bound $U$, Proposition 1 holds based on the monotone convergence theorem.

\section{Interference coordination with dis- tributed algorithm}

As analyzed in Section 2, we recognize that the co-tier interference is incurred only when SBSs are in the same full-duplex modes. Thus, we can determine that there is an intuitive relationship between our problem and the graph coloring problem if we regard these two full-duplex modes as two distinct colors. Based on this idea, in this section, we propose a distributed graph coloring algorithm (DGCA) based on price, which has each SBS individually choose the full-duplex mode, to find a near-optimal solution. As compared with centralized algorithms, DGCA could significantly reduce the computational overhead of MBS.

\subsection{Adjacent graph}

Considering the large-scale fading, the $i$ th SUE associated with the $i$ th SBS suffers from only severe co-tier interference incurred by nearby SBSs that operate in the same mode. To describe this phenomenon, we make the following definition:

Definition 1 The $i$ th SBS and the $j$ th SBS are adjacent if and only if $\eta_{i, j}=1$, where

$$
\eta_{i, j} \triangleq \begin{cases}1, & c_{i, j}>\Gamma_{\mathrm{th}} \text { or } c_{j, i}>\Gamma_{\mathrm{th}} \\ 0, & \text { otherwise }\end{cases}
$$

and $\Gamma_{\mathrm{th}}$ is an interference-to-signal ratio threshold.
Remark 1 Intuitively, $c_{i, j}$ denotes the ratio between the interference from SBS $j$ to SUE $i$ and the useful signal transmitted by SBS $i$ to SUE $i$. As such, when saying that two SBSs are adjacent, we mean that one of them may cause significant co-tier interference to the other's associated SUE. The co-tier interference between nonadjacent SBSs is comparatively small and can be ignored.

Applying this definition to all SBS pairs, an adjacent graph is generated. Inspired by this adjacent graph, if we want to minimize the co-tier interference in the considered HetNet, we should assign adjacent SBSs with different modes as much as possible. If we further regard these two full-duplex modes as two distinct colors, then we need only to color the adjacent graph according to some kind of graph coloring algorithm.

\subsection{Price}

Some graph coloring algorithms have been proposed in previous studies (Brélaz, 1979; Kim and Cho, 2013). However, the incurred cross-tier interference in the macro-cell tier when an SBS is colored with OBFD mode makes our contribution different from the conventional algorithms.

Now that the cross-tier interference is taken into account, it is necessary to determine which type of interference is predominant for each SBS. For this reason, we consider an extreme case and make the following definition:

Definition 2 Let $C_{\text {I }}$ denote the throughput when all SBSs are in IBFD mode, and let $C_{i, \mathrm{O}}$ be the throughput when only the $i$ th SBS operates in OBFD mode. Then the price of the $i$ th SBS is defined as

$$
\xi_{i} \triangleq C_{i, \mathrm{O}}-C_{\mathrm{I}}
$$

Remark 2 When all SBSs are in IBFD mode, only co-tier interference exists. Under this condition, if the $i$ th SBS changes into OBFD mode, then the original co-tier interference incurred by SBS $i$ disappears, but a new cross-tier counterpart is created. As such, $\xi_{i}$ represents the balance between co-tier interference and cross-tier interference induced by the $i$ th SBS. If $\xi_{i}$ is negative and low, it means that SBS $i$ induces severe cross-tier interference and tends to choose the IBFD mode. Conversely, a positive and high $\xi_{i}$ shows that SBS $i$ results in serious co-tier interference, and it is better to operate in a different mode than its adjacent SBSs. 


\subsection{Distributed graph coloring algorithm}

Capitalizing on the adjacent graph and price defined above, we are now ready to present our DGCA method. Without loss of generality, we restrict our attention to the $i$ th SBS.

As illustrated in Fig. 3, SBS $i$ collects the information of its adjacent SBSs, i.e., full-duplex modes and prices, and divides them into two categories based on their full-duplex modes. For $\operatorname{SBS} j$, which belongs to these two categories, we define the combined price of SBS $i$ and SBS $j$ as follows:

Definition 3 For SBS $i$ and its adjacent $\operatorname{SBS} j$, their combined price is

$$
\xi_{i, j} \triangleq \begin{cases}\xi_{i}+\xi_{j}, & x_{i}=x_{j}=1, \\ \omega \cdot 2^{-\xi_{i}}, & x_{i}=0, x_{j}=1, \\ \omega \cdot 2^{-\xi_{j}}, & x_{i}=1, x_{j}=0, \\ \xi_{i}+\xi_{j}+\omega \cdot 2^{-\xi_{i}}+\omega \cdot 2^{-\xi_{j}}, & x_{i}=x_{j}=0,\end{cases}
$$

where $\omega$ is a weighting factor for cross-tier interference.

Remark 3 When both SBS $i$ and SBS $j$ operate in IBFD mode, it is trivial that their combined price is the summation of the individual prices. However, if they are in different modes, co-tier interference no longer exists but one of them is bound to create cross-tier interference. Without loss of generality, we employ a generalized function that decreases with respect to $\xi$ to describe this combined price. $\omega$ is used as a weighting factor to represent how much crosstier interference will impact the considered system; e.g., $\omega=0$ means that the influence of cross-tier interference can be ignored. Finally, if both SBSs work in OBFD mode, then the individual prices as well as the extra price induced by cross-tier interference need to be considered.

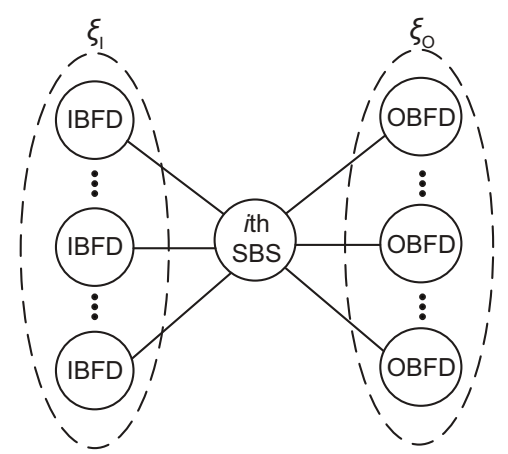

Fig. 3 Schematic diagram for the distributed graph coloring algorithm
Extending this definition to the whole HetNet, the following proposition becomes straightforward:

Proposition 2 The sum price of SBS $i$ and all its adjacent SBSs is

$\xi_{\mathrm{sum}, i}=\left\{\begin{array}{lc}\xi_{\mathrm{I}}+\xi_{i}+\sum_{l \in \mathcal{S}_{\mathrm{O}, i}^{\mathrm{adj}} \omega \cdot 2^{-\xi_{l}},} & x_{i}=1, \xi_{\mathrm{I}} \neq 0, \\ \sum_{l \in \mathcal{S}_{\mathrm{O}, i}^{\mathrm{adj}} \omega \cdot 2^{-\xi_{l}},} & x_{i}=1, \xi_{\mathrm{I}}=0, \\ \xi_{\mathrm{O}}+\xi_{i}+\sum_{l \in \mathcal{S}_{\mathrm{O}, i}^{\mathrm{adj}} \omega \cdot 2^{-\xi_{l}}+\omega} \cdot 2^{-\xi_{i}}, & x_{i}=0, \xi_{\mathrm{O}} \neq 0, \\ \sum_{l \in \mathcal{S}_{\mathrm{O}, i}^{\text {adj }} \omega \cdot 2^{-\xi_{l}}+\omega \cdot 2^{-\xi_{i}},} & x_{i}=0, \xi_{\mathrm{O}}=0,\end{array}\right.$

where $\xi_{\mathrm{I}}=\sum_{l \in \mathcal{S}_{\mathrm{I}, i}^{\text {adj }}} \xi_{l}, \mathcal{S}_{\mathrm{I}, i}^{\text {adj }} \triangleq\left\{j \mid \boldsymbol{x}_{j}=1, \eta_{i, j}=1\right\}$, $\xi_{\mathrm{O}}=\sum_{l \in \mathcal{S}_{\mathrm{O}, i}^{\text {adj }}} \xi_{l}$, and $\mathcal{S}_{\mathrm{O}, i}^{\mathrm{adj}} \triangleq\left\{j \mid \boldsymbol{x}_{j}=0, \eta_{i, j}=1\right\}$.

Now we have the sum price of SBS $i$ to the whole HetNet. To minimize the sum price, Proposition 3 can be easily obtained, if we compare the sum prices when SBS $i$ chooses a different full-duplex mode.

Proposition 3 The $i$ th SBS should select the full-duplex mode with the following rules:

Case 1 If $\xi_{\mathrm{I}}=0$, then $x_{i}=1$.

Case 2 If $\xi_{\mathrm{I}} \neq 0, \xi_{\mathrm{O}} \neq 0$, then

$$
x_{i}= \begin{cases}1, & \xi_{\mathrm{I}}<\xi_{\mathrm{O}}+\omega \cdot 2^{-\xi_{i}}, \\ 0, & \text { otherwise. }\end{cases}
$$

Case 3 If $\xi_{\mathrm{I}} \neq 0, \xi_{\mathrm{O}}=0$, then

$$
x_{i}= \begin{cases}1, & \xi_{\mathrm{I}}+\xi_{i}<\omega \cdot 2^{-\xi_{i}}, \\ 0, & \text { otherwise }\end{cases}
$$

Based on the results above, the specific DGCA is shown in Algorithm 3. We can see that the computational overhead of MBS could be significantly reduced with SBSs affording plenty of computation in DGCA. Notably, we sort the SBSs by price in ascending order in step 2. This is because DGCA tends to choose IBFD mode for the first few SBSs due to small $\xi_{\mathrm{I}}$ and $\xi_{\mathrm{O}}$, and in the meantime, the network benefits from assigning low price SBSs with IBFD mode. Moreover, there exist two parameters $\Gamma_{\text {th }}$ and $\omega$ in DGCA, which play an important role in our algorithm. They may have different influences on the performance of DGCA in different scenarios, i.e., dense or sparse small-cell networks; thus, in our algorithm we assess a range of $\Gamma_{\text {th }}$ and $\omega$ to choose the optimal $\Gamma_{\text {th }}$ and $\omega$.

As for the analysis of complexity, we can see that in the phases of obtaining adjacent graphs and 


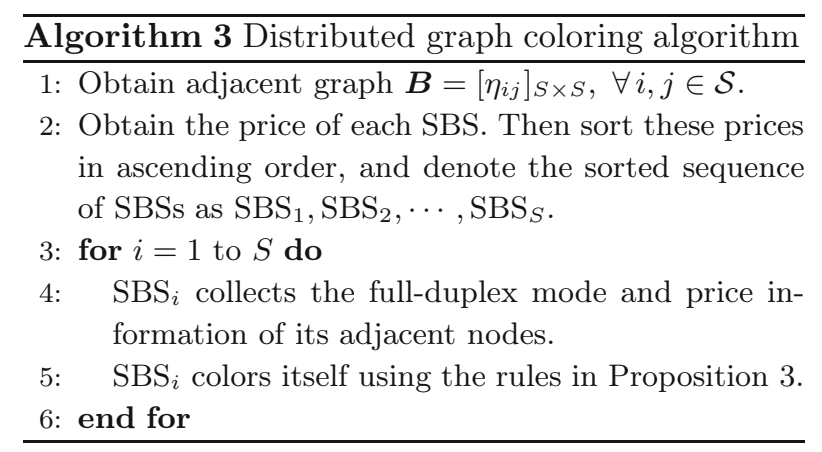

distributed coloring, i.e., step 1 and steps $3-6$, the complexity is $\mathcal{O}\left(S^{2}\right)$; however, in the phase of obtaining the price of each SBS, i.e., step 2, the complexity is $\mathcal{O}\left(S^{3}\right)$, which makes the overall complexity of DGCA grow. Fortunately, step 2 needs only to be executed once in DGCA, and thus the computational complexity of DGCA will be less than those of GEA and GRA, which execute high-complexity steps repeatedly.

\section{Performance evaluation}

In this section, various numerical simulations are presented to evaluate the performance of the proposed algorithms. We consider a circular cell of radius $R_{\mathrm{M}}$ where the MBS is located at the center, and $K$ MUEs and $S$ SBSs are uniformly scattered within the cell region. Each individual SUE is randomly placed within the circle of radius $R_{\mathrm{S}}$ from its associated SBS. The channel gain has a small-scale Rayleigh fading component and a large-scale fading component. To exploit the advantages of multiple antennas, we consider the non-line-of-sight (NLOS) model provided in $3 \mathrm{GPP}$ (2012b) as the large-scale fading including path loss and shadowing fading. The parameters used in the simulations are listed in Table 1. Notably, the system suffers from the most serious co-tier interference and cross-tier interference when all SBSs operate in OBFD mode, and the induced throughput can be seen as a lower bound (LB). We take this LB as the benchmark to compare the performances of the proposed algorithms.

Fig. 4 shows the downlink throughput obtained by GEA, DGCA, and GRA. For all these algorithms, the downlink throughput increases almost linearly with the increase of the number of SBSs. This is intuitive because a dense small-cell network leads to better network performance. Also, it can be seen that GEA, DGCA, and GRA all induce a significant
Table 1 List of simulation parameters

\begin{tabular}{lc}
\hline \multicolumn{1}{c}{ Parameter } & Value \\
\hline Macro-cell radius, $R_{\mathrm{M}}$ & $1000 \mathrm{~m}$ \\
Small-cell radius, $R_{\mathrm{S}}$ & $40 \mathrm{~m}$ \\
Carrier frequency, $f_{\mathrm{c}}$ & $2 \mathrm{GHz}$ \\
System bandwidth, $B$ & $20 \mathrm{MHz}$ \\
Number of MUEs, $K$ & 20 \\
Transmit power of $\mathrm{MBS}, P_{\mathrm{M}}$ & $46 \mathrm{dBm}$ \\
Transmit power of SBS, $P_{\mathrm{S}}$ & $24 \mathrm{dBm}$ \\
Noise power, $\sigma^{2}$ & $-174 \mathrm{dBm} / \mathrm{Hz}$ \\
\hline
\end{tabular}

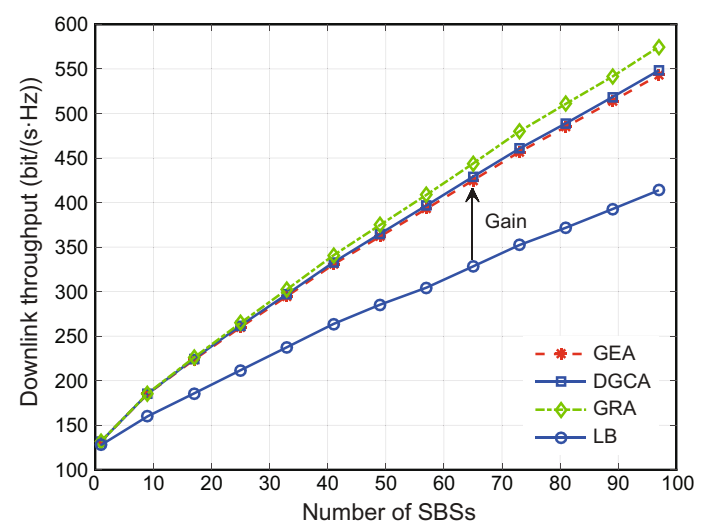

Fig. 4 Downlink capacity versus the number of SBSs when the number of transmit antennas in MBS is 200

gain compared with the LB benchmark, which verifies the efficiency of the proposed algorithms. In a comparison of the three algorithms, GRA has the best performance. Notably, as a distributed algorithm, DGCA is very promising because its performance is the closest to that of GEA.

Next, Fig. 5 shows the mode selection proportion of IBFD with respect to the number of SBSs. We see that as the number of SBSs increases, the proportion decreases gradually. The reason is that the co-tier interference becomes rather severe in dense networks and it is better to assign SBSs with different modes as much as possible. It can be inferred that the optimal proportion tends to be 0.5 in ultradense small-cell networks.

In Figs. 6 and 7, we investigate how the number of MBS antennas influences the considered HetNet. Fig. 6 shows that more MBS antennas induce higher downlink throughput, but the growth is slow. Fig. 7 shows that the selection proportion hardly changes with the increase of the number of MBS antennas. This is reasonable because massive MIMO impacts only the macro-cell tier in problem (16) and has little influence on the interference in a small-cell tier. 


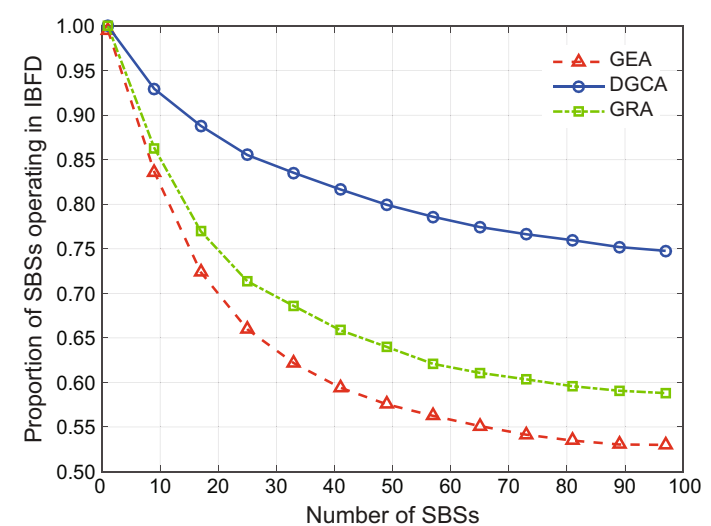

Fig. 5 Selection proportion of IBFD mode versus the number of SBSs when the number of transmit antennas in MBS is 200

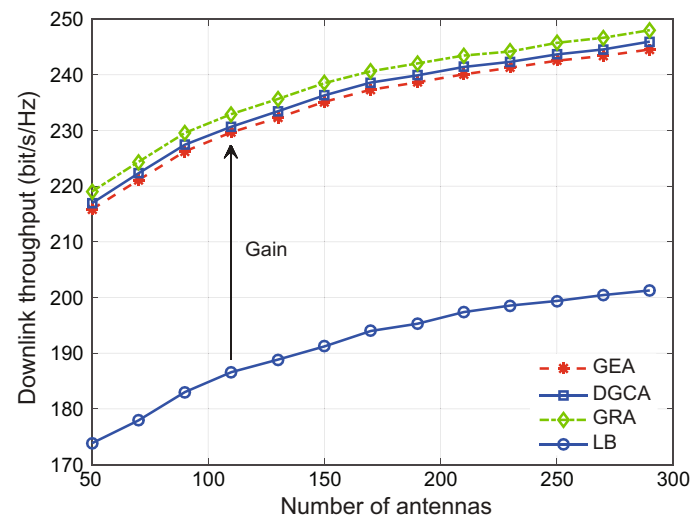

Fig. 6 Downlink capacity versus the number of antennas $N$ when the number of single-antenna SBSs is 20

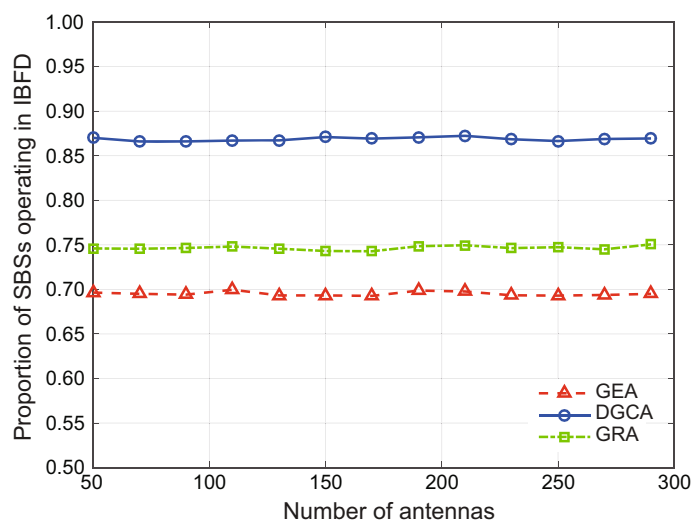

Fig. 7 Selection proportion of IBFD mode versus the number of antennas $N$ when the number of singleantenna SBSs is 20

Fig. 8 shows the optimal $\Gamma_{\text {th }}$ and $\omega$ of DGCA versus the number of SBSs. The optimal $\Gamma_{\text {th }}$ increases gradually with an increase in the number of SBSs. This is because there are many unnecessary nodes, which make the solution imprecise in dense

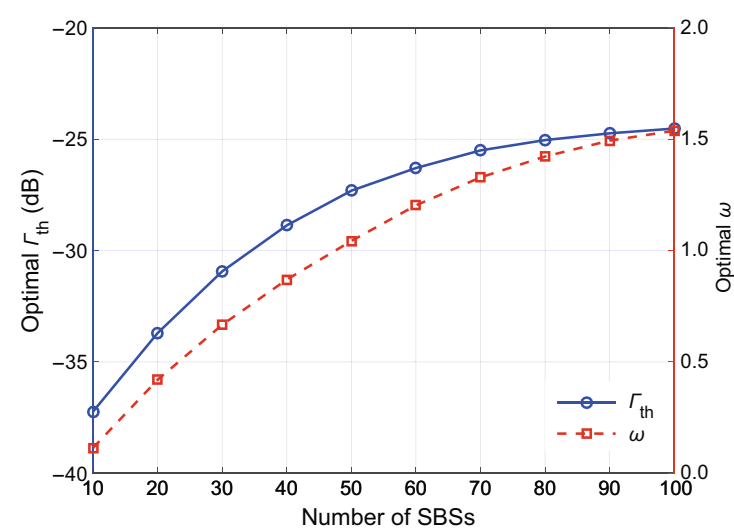

Fig. 8 The optimal $\Gamma_{\text {th }}$ and $\omega$ of DGCA versus the number of SBSs when the number of transmit antennas in MBS is 200

networks, and thus we need to increase the threshold. $\omega$ has a similar curve, because the macro-cell tier will suffer from a lot of cross-tier interference from SBSs as the network becomes denser, and cross-tier interference should play a more important part in the HetNet.

We further investigate the computation time of the proposed algorithms (Fig. 9). Although we have proved that the complexity of GEA, DGCA, and each iteration in GRA is identical, the difference in their computation time needs to be further investigated. Fig. 9 shows that the computation time of GRA is less than that of GEA when $S$ is small, but grows rapidly as $S$ increases. This indicates that the convergence rate of GRA drops quickly with respect to $S$. Also, we see that DGCA has the least computation time. It seems that DGCA has linear complexity when $S$ is small, yet its complexity is exponential in fact. As such, we conclude that for

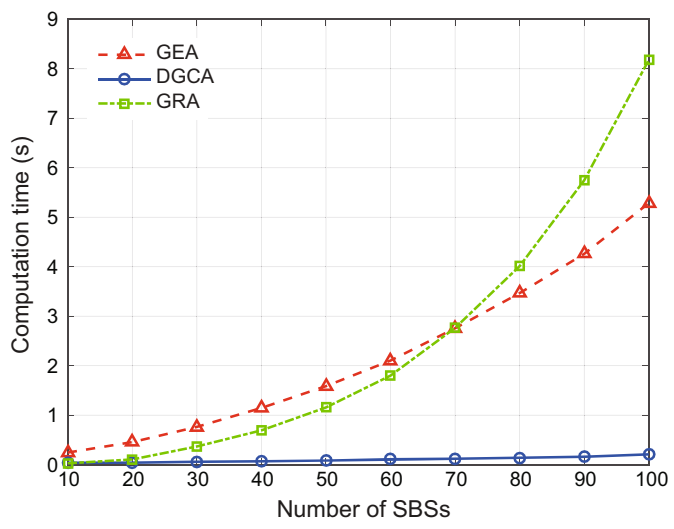

Fig. 9 CPU time of the proposed algorithms versus the number of SBSs when the number of transmit antennas in MBS is 200 
centralized algorithms, GRA has higher computation time than GEA but has the best throughput performance, and DGCA, as a distributed algorithm, has the least computation time and similar throughput performance to centralized algorithms.

\section{Conclusions}

In this paper, focusing on the downlink of a twotier HetNet that incorporates massive MIMO employed in MBS and full-duplex employed in SBSs, an interference coordination framework is described and analyzed. To suppress the network interference and maximize the downlink throughput, two centralized algorithms, i.e., a genetic algorithm and a greedy algorithm, are proposed to decide the fullduplex mode selection strategies of SBSs. In particular, a distributed graph coloring algorithm, in which each SBS individually chooses the full-duplex mode, is further proposed to reduce the computation overhead of the MBS. Numerical results show that the proposed algorithms have significant performance gain compared with the benchmark, and there exists a trade-off among the throughput performance, computation time, and computation overhead of the MBS when we estimate these three algorithms.

\section{References}

3GPP, 2012a. Evolved universal terrestrial radio access (EUTRA); LTE physical layer; general description. Technical Specification No. 36.201 (v11.1.0), 3rd Generation Partnership Project.

3GPP, 2012b. Evolved universal terrestrial radio access (E-UTRA); further enhancements to LTE time division duplex (TDD) for downlink-uplink (DL-UL) interference management and traffic adaptation. Technical Report No. 36.828 (v11.0.0), 3rd Generation Partnership Project.

Bharadia, D., Katti, S., 2016. Full-duplex radios. In: Vannithamby, R., Talwar, S. (Eds.), Towards 5G: Applications, Requirements and Candidate Technologies. John Wiley \& Sons, p.365-394.

http://dx.doi.org/10.1002/9781118979846.ch16

Boccardi, F., Heath, R., Lozano, A., et al., 2014. Five disruptive technology directions for 5G. IEEE Commun. Mag., 52(2):74-80.

http://dx.doi.org/10.1109/mcom.2014.6736746

Brélaz, D., 1979. New methods to color the vertices of a graph. Commun. ACM, 22(4):251-256. http://dx.doi.org/10.1145/359094.359101

Choi, J.I., Jain, M., Srinivasan, K., et al., 2010. Achieving single channel, full duplex wireless communication. 16th Annual Int. Conf. on Mobile Computing and Networking, p.1-12.

http://dx.doi.org/10.1145/1859995.1859997
Goyal, S., Liu, P., Hua, S., et al., 2013. Analyzing a full-duplex cellular system. 47th Annual Conf. on Information Sciences and Systems, p.1-6. http://dx.doi.org/10.1109/ciss.2013.6552310

Goyal, S., Liu, P., Panwar, S., et al., 2014. Improving small cell capacity with common-carrier full duplex radios. IEEE Int. Conf. on Communications, p.4987-4993. http://dx.doi.org/10.1109/icc.2014.6884111

Hosseini, K., Hoydis, J., ten Brink, S., et al., 2013. Massive MIMO and small cells: how to densify heterogeneous networks. IEEE Int. Conf. on Communications, p.5442-5447. http://dx.doi.org/10.1109/icc.2013.6655455

Hoydis, J., Kobayashi, M., Debbah, M., 2011. Green smallcell networks. IEEE Veh. Technol. Mag., 6(1):37-43. http://dx.doi.org/10.1109/mvt.2010.939904

Hoydis, J., Hosseini, K., ten Brink, S., et al., 2013. Making smart use of excess antennas: massive MIMO, small cells, and TDD. Bell Labs Techn. J., 18(2):5-21. http://dx.doi.org/10.1002/bltj.21602

Jain, M., Choi, J.I., Kim, T., et al., 2011. Practical, real-time, full duplex wireless. 17th Annual Int. Conf. on Mobile Computing and Networking, p.301312. http://dx.doi.org/10.1145/2030613.2030647

Kim, S., Cho, I., 2013. Graph-based dynamic channel assignment scheme for femtocell networks. IEEE Commun. Lett., $\mathbf{1 7}(9): 1718-1721$.

http://dx.doi.org/10.1109/lcomm.2013.071013.130585

Larsson, E., Edfors, O., Tufvesson, F., et al., 2014. Massive MIMO for next generation wireless systems. IEEE Commun. Mag., 52(2):186-195. http://dx.doi.org/10.1109/mcom.2014.6736761

Li, B., Zhu, D., Liang, P., 2015. Small cell in-band wireless backhaul in massive MIMO systems: a cooperation of next-generation techniques. IEEE Trans. Wirel. Commun., 14(12):7057-7069.

http://dx.doi.org/10.1109/twc.2015.2464299

Liu, G., Yu, F.R., Ji, H., et al., 2015. In-band full-duplex relaying: a survey, research issues and challenges. IEEE Commun. Surv. Tutor., 17(2):500-524. http://dx.doi.org/10.1109/comst.2015.2394324

Marzetta, T.L., 2010. Noncooperative cellular wireless with unlimited numbers of base station antennas. IEEE Trans. Wirel. Commun., 9(11):3590-3600. http://dx.doi.org/10.1109/twc.2010.092810.091092

Rusek, F., Persson, D., Lau, B.K., et al., 2013. Scaling up MIMO: opportunities and challenges with very large arrays. IEEE Signal Process. Mag., 30(1):40-60. http://dx.doi.org/10.1109/msp.2011.2178495

Sabharwal, A., Schniter, P., Guo, D., et al., 2014. Inband full-duplex wireless: challenges and opportunities. IEEE J. Sel. Areas Commun., 32(9):1637-1652. http://dx.doi.org/10.1109/jsac.2014.2330193

Tabassum, H., Sakr, A.H., Hossain, E., 2016. Analysis of massive MIMO-enabled downlink wireless backhauling for full-duplex small cells. IEEE Trans. Commun., 64(6):2354-2369. http://dx.doi.org/10.1109/tcomm.2016.2555908

Thilina, K.M., Tabassum, H., Hossain, E., et al., 2015. Medium access control design for full duplex wireless systems: challenges and approaches. IEEE Commun. Mag., 53(5):112-120. http://dx.doi.org/10.1109/mcom.2015.7105649 